\title{
High-Resolution Two-Dimensional Optical Spectroscopy of Electron Spins
}

\author{
M. Salewski, ${ }^{1}$ S. V. Poltavtsev, ${ }^{1,2}$ I. A. Yugova, ${ }^{2}$ G. Karczewski, ${ }^{3}$ M. Wiater, ${ }^{3}$ T. Wojtowicz, ${ }^{3,4}$ \\ D. R. Yakovlev, ${ }^{1,5}$ I. A. Akimov, ${ }^{1,5}$ T. Meier, ${ }^{6}$ and M. Bayer ${ }^{1,5}$ \\ ${ }^{1}$ Experimentelle Physik 2, Technische Universität Dortmund, 44221 Dortmund, Germany \\ ${ }^{2}$ Spin Optics Laboratory, St. Petersburg State University, St. Petersburg 198504, Russia \\ ${ }^{3}$ Institute of Physics, Polish Academy of Sciences, PL-02668 Warsaw, Poland \\ ${ }^{4}$ International Research Centre MagTop, PL-02668 Warsaw, Poland \\ ${ }^{5}$ Ioffe Institute, Russian Academy of Sciences, 194021 St. Petersburg, Russia \\ ${ }^{6}$ Department Physik \& CeOPP, Universität Paderborn, D-33098 Paderborn, Germany \\ (Received 2 January 2017; revised manuscript received 15 May 2017; published 14 August 2017)
}

\begin{abstract}
Multidimensional coherent optical spectroscopy is one of the most powerful tools for investigating complex quantum mechanical systems. While it was conceived decades ago in magnetic resonance spectroscopy using microwaves and radio waves, it has recently been extended into the visible and UV spectral range. However, resolving $\mathrm{MHz}$ energy splittings with ultrashort laser pulses still remains a challenge. Here, we analyze two-dimensional Fourier spectra for resonant optical excitation of resident electrons to localized trions or donor-bound excitons in semiconductor nanostructures subject to a transverse magnetic field. Particular attention is devoted to Raman coherence spectra, which allow one to accurately evaluate tiny splittings of the electron ground state and to determine the relaxation times in the electron spin ensemble. A stimulated steplike Raman process induced by a sequence of two laser pulses creates a coherent superposition of the ground-state doublet which can be retrieved only optically because of selective excitation of the same subensemble with a third pulse. This provides the unique opportunity to distinguish between different complexes that are closely spaced in energy in an ensemble. The related experimental demonstration is based on photon-echo measurements in an $n$-type $\mathrm{CdTe} /(\mathrm{Cd}, \mathrm{Mg}) \mathrm{Te}$ quantum-well structure detected by a heterodyne technique. The difference in the sub- $\mu \mathrm{eV}$ range between the Zeeman splittings of donor-bound electrons and electrons localized at potential fluctuations can be resolved even though the homogeneous linewidth of the optical transitions is larger by 2 orders of magnitude.
\end{abstract}

DOI: 10.1103/PhysRevX.7.031030

\section{INTRODUCTION}

Coherent optical spectroscopy has been widely used for the investigation of the energy levels of charge, vibration, and spin states in condensed matter systems [1,2]. It comprises many elaborated techniques that can be roughly divided into two main categories: The first set uses continuous wave light sources of tunable wavelength with narrow spectral width, i.e., long coherence time, which enable direct acquisition of coherent spectra in the frequency domain [3]. The second set is based on recording the temporal dynamics of the system's response to short light pulse excitation [4]. Here, Fourier transformation of the optical transients into the frequency domain allows one to obtain similar information, not only about the energy level structure but also about the inhomogeneous and homogeneous widths of the involved optical transitions as well as the coherent dynamics of the system

Published by the American Physical Society under the terms of the Creative Commons Attribution 4.0 International license. Further distribution of this work must maintain attribution to the author(s) and the published article's title, journal citation, and DOI.
Subject Areas: Condensed Matter Physics,

Materials Science, Optics under study. One of these techniques is the two-dimensional Fourier transform spectroscopy (2DFTS), which is based on the Fourier transformation of transient four-wave mixing (FWM) signals. This technique has rapidly developed during the last decade and has been successfully applied for the investigation of atomic, molecular, and condensed matter systems such as organic and inorganic semiconductors [5-9]. One of the appealing features of 2DFTS is the intuitive visualization of the underlying physics, as it not only enables one to extract energy levels but also provides a clear understanding of the dynamics and correlations between optical excitations [10].

Systems with more than two states interacting with light attract particular interest. The most representative examples are $\mathrm{V}$-type ( $\Lambda$-type) energy level orders where the single ground (excited) state is optically coupled to an excited (ground) state doublet [11]. These level schemes make it possible to observe several fascinating phenomena such as quantum beats, coherent population trapping, and electromagnetically induced transparency (EIT), which may be used for applications in quantum information technology $[3,12]$. A main feature of a $\Lambda$ scheme is the possibly long coherence time of the ground states. In semiconductors, the 
$\Lambda$ scheme can be obtained for optical excitation of localized excess carriers, e.g., electrons in the conduction band or holes in the valence band, using their spin degree of freedom [13]. In the case of resident electrons, the negatively charged exciton (trion $X^{-}$) and the donor-bound exciton $\left(D^{0} X\right)$ are possible optically excited states. Coherent population trapping and EIT have been demonstrated for donor-bound excitons in bulk GaAs [14,15], as well as trions in $n$-type CdTe quantum wells (QWs) [16] and quantum dots (QDs) charged with resident carriers [17-19]. These studies have exploited high-resolution spectroscopy with continuous-wave lasers. Spin control of resident carriers using ultrafast laser pulses has also been successfully demonstrated [20-22]. However, most of the FWM and 2DFTS experiments on charged excitations in semiconductors have not exploited the spin degree of freedom in the ground state so far [23-26]. Recently, we demonstrated that the ground-state splitting of a trion in a transverse magnetic field leads to quantum beats in the photon echoes at the Larmor precession frequency [27]. This allowed us to perform a coherent transfer of optical excitation into a spin ensemble and to observe long-lived photon echoes [28]. Here, we demonstrate that our approach can be used as a tool for spectroscopy of the ground-state levels with remarkably high resolution: In an $n$-type $\mathrm{CdTe} /(\mathrm{Cd}, \mathrm{Mg}) \mathrm{Te} \mathrm{QW}$, we are able to resolve splittings between the spin sublevels with sub- $\mu \mathrm{eV}$ precision and to distinguish between different types of electrons in the ensemble, namely, electrons either bound to donors or localized on QW potential fluctuations. To that end, we show that stimulated steplike Raman processes in the two-pulse excitation scheme allow us to probe the electron spin ensemble with high selectivity and precision even for systems with broad optical transitions, given by large inhomogeneous broadening due to system variations or short optical coherence times leading to strong homogeneous broadening. Therefore, our approach using ultrashort optical pulses mimics EIT, which typically requires frequencystabilized lasers to resolve splittings of that magnitude.

The paper is organized as follows. First, we consider theoretically 2DFTS spectra under resonant excitation of localized trions or donor-bound excitons subject to a transverse magnetic field. We focus on Raman coherence spectra, which allow us to evaluate the splitting of the ground state and to determine the relaxation times in the electron spin ensemble. Second, we show experimental results on heterodyne-detected photon echoes recorded for a $\mathrm{CdTe} /(\mathrm{Cd}, \mathrm{Mg}) \mathrm{Te} \mathrm{QW}$ in which both trions and donorbound exciton states are present.

\section{TWO-DIMENSIONAL FOURIER SPECTRA IN NANOSTRUCTURES WITH RESIDENT ELECTRONS}

We concentrate on optical transitions in zinc-blende semiconductor nanostructures with resident electrons in the conduction band. In that way, a $\Lambda$-transition scheme with long-lived coherence in the ground state can be established. We consider type-I semiconductor QWs with the heavy and light holes split by the confinement potential along the $z$ direction. At cryogenic temperatures, the resident electrons are localized on potential fluctuations. Alternately, the electrons can become trapped by donors. We consider an electron ensemble of low density, where the electrons are well separated and do not interact with each other. The ground state is a doublet with electron spin $S=1 / 2$. The lowest-energy, almost-degenerate states that can be optically excited are the localized trion or the donor-bound exciton, whose angular momentum $J=3 / 2$ is given by the heavy hole. Higher-energy states, e.g., the neutral exciton or the light-hole exciton transitions, can be neglected if the optical pulses used for excitation are spectrally narrow enough. In a transverse magnetic field $B$ applied along the $x$ direction, the electron spin states are split by $\hbar \omega_{L}=g \mu_{B} B$, where $\omega_{L}$ is the Larmor precession frequency, $g$ is the electron $g$ factor, and $\mu_{B}$ is the Bohr magneton. Optical transitions between all four states are allowed using light with linear polarization directed along $(\mathrm{H})$ or perpendicular to $(\mathrm{V})$ the direction of the magnetic field $(\mathrm{H} \| x$ and $\mathrm{V} \| y)$. The energy level structure and optical transitions are shown in Fig. 1(b). The studied system can be considered as composed of two $\Lambda$ schemes sharing common ground states. This energy level structure is realized in a large variety of atomic and solid-state objects with pseudospin in the ground and excited states [11].

Transient FWM requires three optical pulses with wave vectors $\mathbf{k}_{1}, \mathbf{k}_{2}$, and $\mathbf{k}_{3}$, respectively, separated in time by $t_{1}, t_{2}$. The time $t_{3}$ gives the temporal delay of the resulting FWM signal with respect to pulse 3 [see Fig. 1(a)]. Because of the inhomogeneous broadening of the optical transitions, the transient FWM signal is considered in the rephasing configuration, and the resulting photon echoes are described by the optical field

$$
S_{I}\left(t_{1}, t_{2}, t_{3}\right)=A_{\mathrm{PE}}\left(t_{1}, t_{2}, t_{3}\right) e^{-\left[\left(t_{3}-t_{1}\right)^{2} / 2 \sigma^{2}\right]} e^{i \omega\left(t_{3}-t_{1}\right)},
$$

where $\sigma$ is the degree of (Gaussian) inhomogeneity and $\omega$ is the central frequency of the optical pulses, which are tuned in resonance with the central frequency of the inhomogeneous ensemble [4,5]. The amplitude of the photon echo $A_{\mathrm{PE}}\left(t_{1}, t_{2}, t_{3}\right)$ depends on the delay times $t_{1}, t_{2}$, and $t_{3}$ as well as on the polarization configuration of the exciting pulses. A proper polarization choice in the pulse sequence provides additional selectivity between various excitation paths [28]. This can be traced from the double-sided Feynman diagrams that are shown in Figs. 1(c) and 1(d). The entirety of possible polarization configurations can be found in the supplementary material [29]. Here, we consider the HHH (VVV) and HVV (VHH) polarization sequences, which correspond to the most representative cases. Following the Feynman diagrams, it is seen that the 
(a)

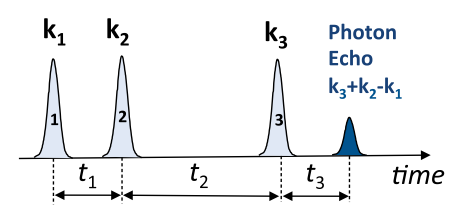

(b)

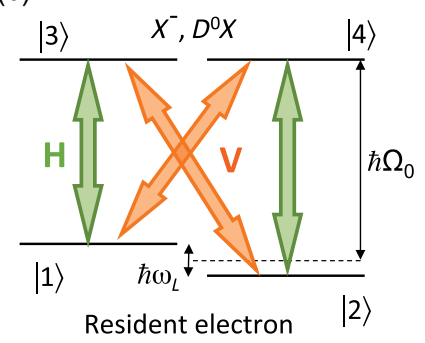

(c)

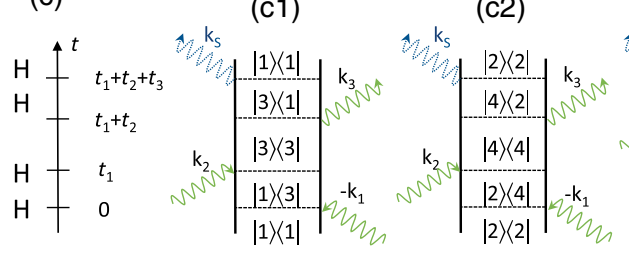

(c3) (c4)

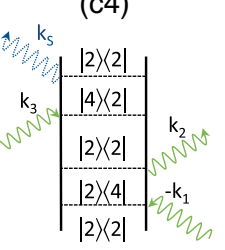

(d)

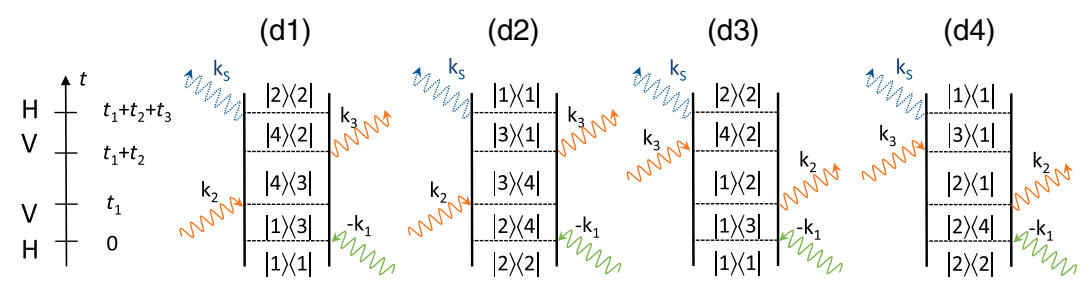

FIG. 1. (a) Scheme of the pulse sequence and the photon-echo signal that is detected in the $\mathbf{k}_{S}=\mathbf{k}_{3}+\mathbf{k}_{2}-\mathbf{k}_{1}$ direction. (b) Energylevel diagram and optical transitions for the trion $\left(X^{-}\right)$or the donor-bound exciton $\left(D^{0} X\right)$, which are localized in the semiconductor QW structure. The characters correspond to the polarization of the optical transition parallel $(\mathrm{H})$ and perpendicular $(\mathrm{V})$ to the magnetic field. (c,d) Double-sided Feynman diagrams for the HHH and HVV polarization configurations, respectively. For VVV and VHH, the resulting diagrams are identical to HHH and HVV if the states $|3\rangle$ and $|4\rangle$ are exchanged. For the full set of diagrams, see also Sec. II of the supplementing material [29].

resulting photon echo is $\mathrm{H}(\mathrm{V})$ polarized. For $\mathrm{HHH}$, the possible optical transitions take place between the two independent two-level pairs $|1\rangle-|3\rangle$ and $|2\rangle-|4\rangle$, while for HVV all transitions are involved, and the coherent superposition between one pair of states is transferred to the other pair after each excitation event in a steplike process.

The first H-polarized pulse addresses the two optical transitions between $|1\rangle$ and $|3\rangle$ at frequency $\Omega_{0}-\omega_{L} / 2$ and between $|2\rangle$ and $|4\rangle$ at frequency $\Omega_{0}+\omega_{L} / 2$. Here, $\Omega_{0}$ corresponds to the optical resonance frequency at zero magnetic field $\left(\omega_{L}=0\right)$. Pulse 1 creates two independent coherent superpositions between the pairs of states $|1\rangle-|3\rangle$ and $|2\rangle-|4\rangle$, which can be considered as optical coherences. In the density matrix formalism, they correspond to the $\rho_{13}$ and $\rho_{24}$ elements of the density matrix, respectively. Here, we assume that before excitation with pulse 1 , the system is in the ground state, and the only nonzero density matrix elements are $\rho_{11}=\rho_{22}=1 / 2$, i.e., $\hbar \omega_{L} \ll k_{B} T$, where $k_{B}$ is the Boltzmann constant and $T$ is the temperature. The second and third pulses are both $\mathrm{H}$ or $\mathrm{V}$ polarized. Possible quantum mechanical pathways for the evolution of the system follow from the double-sided Feynman diagrams in Figs. 1(c) and 1(d), respectively.

(i) HHH copolarized configuration. The second pulse addresses the same pairs of optical transitions and, in that way, the optical coherences $\rho_{13}$ and $\rho_{24}$ are transformed into the excited-state populations $\rho_{33}$ and $\rho_{44}$ [diagrams (c1) and (c2) in Fig. 1(c)] as well as the ground-state populations $\rho_{11}$ and $\rho_{22}$ [diagrams (c3) and (c4) in Fig. 1(c)]. The populations carry the information about the optical phase $\phi_{ \pm}=\left(\Omega_{0} \pm \omega_{L} / 2\right) t_{1}$ between the pulses 1 and 2, i.e., $\rho_{33} \propto \sin ^{2}\left(\phi_{-} / 2\right)$ and $\rho_{44} \propto \sin ^{2}\left(\phi_{+} / 2\right)$, where $\rho_{11}+\rho_{33}=\rho_{22}+\rho_{44}=1 / 2$ holds. The third pulse induces the coherences $\rho_{31}$ and $\rho_{42}$ and results in the emission of the photon echo after the rephasing process. At zero magnetic field, the excited-state populations are identical, $\rho_{33}=\rho_{44}$, and the dynamics are determined by the decay of the trion or donor-bound exciton complex when $t_{2}$ is varied. However, when the magnetic field is applied, $\rho_{33}-\rho_{44} \propto \sin \left(\omega_{L} t_{1} / 2\right) \sin \left(\Omega_{0} t_{1}\right)$; i.e., for a given $\Omega_{0}$, there are nonzero spin populations $J_{x}=\left(\rho_{33}-\right.$ $\left.\rho_{44}\right) / 2$ and $S_{x}=\left(\rho_{11}-\rho_{22}\right) / 2=-J_{x}$ in the excited and ground states, respectively. The spin populations carry the information about $\phi_{ \pm}$and correspondingly contribute to the coherent optical response [28].

(ii) HVV cross-polarized configuration. Here, the second pulse accomplishes a stimulated steplike Raman process, where the optical coherences $\rho_{13}$ and $\rho_{24}$ are transferred into the $X^{-}$or $D^{0} X$ spin coherence $\rho_{34}$ [see diagrams (d1) and (d2) in Fig. 1(d)] and the electron spin coherence $\rho_{12}$ [see diagrams (d3) and (d4) in Fig. 1(d)]. The third pulse induces a back transformation of the trion and electron spin coherences into the optical coherences $\rho_{42}$ and $\rho_{31}$. This mechanism exploits off-diagonal density matrix elements. Thereby, the Raman process initiates a shift of the optical frequency of the emitted signal by $+\omega_{L}$ or $-\omega_{L}$ when starting from $\rho_{11}$ or $\rho_{22}$, respectively. 
For the case in which the splitting of the electron spin sublevels in the ground state is smaller than the spectral width of the excitation laser pulses $\left(t_{p} \omega_{L} \ll 2 \pi\right.$, where $t_{p}$ is the pulse duration) and the inhomogeneous broadening $\left(\omega_{L} \sigma \ll 1\right)$, the echo signals can be well approximated by Gaussian pulses with an amplitude $A_{\mathrm{PE}}\left(t_{1}, t_{2}\right)$ that depends on $t_{1}$ and $t_{2}$ only. In the linear copolarized polarization configuration,

$$
\begin{aligned}
A_{\mathrm{PE}}^{\|} \propto & e^{-\left[\left(2 t_{1}\right) / T_{2}\right]} e^{-\left(t_{2} / \tau_{r}\right)}\left[2 \cos ^{2}\left(\omega_{L} t_{1} / 2\right)\right. \\
& \left.+e^{-\left(t_{2} / T_{h}\right)} \sin ^{2}\left(\omega_{L} t_{1} / 2\right)\right] \\
& +e^{-\left[\left(2 t_{1}\right) / T_{2}\right]} e^{-\left[t_{2} /\left(T_{1}^{e}\right)\right]} \sin ^{2}\left(\omega_{L} t_{1} / 2\right),
\end{aligned}
$$

where $T_{2}$ and $\tau_{r}$ are the coherence time and the lifetime of the optically excited $X^{-}$or $D^{0} X$ complex, $T_{h}$ is the spin relaxation time of the hole for $X^{-}$or $D^{0} X$, and $T_{1}^{e}$ is the longitudinal spin relaxation time of the electron in the ground state [29]. The first term on the right-hand side of Eq. (2) represents the population decay of the photoexcited complex (negative trion or donor-bound exciton) due to spontaneous recombination. The second and third terms originate from the spin relaxation of the hole in the excited state and the electron in the ground state, respectively. Note that the decay time of the last term is governed only by the spin relaxation time $T_{1}^{e}$ and represents the longlived ground-state spin population $S_{x}$ (for more details, see also the Supplementary Material [29]). For $t_{2}=0$, the signal is

$$
A_{\mathrm{PE}, \mathrm{t}_{2}=0}^{\|} \propto e^{-\left[\left(2 t_{1}\right) / T_{2}\right]},
$$

which is independent of the magnetic field.

In the cross-polarized linear polarization configuration, the signal is given by

$$
\begin{aligned}
A_{\mathrm{PE}}^{\perp} \propto & e^{-\left[\left(2 t_{1}\right) / T_{2}\right]\left[e^{-\left(t_{2} / \tau_{T}\right)} \cos \left(\omega_{L} t_{1}\right)\right.} \\
& \left.+e^{-\left[t_{2} /\left(T_{2}^{e}\right)\right]} \cos \left(\omega_{L}\left(t_{1}+t_{2}\right)\right)\right],
\end{aligned}
$$

if we assume that $\omega_{L} t_{1} \gg 2 \pi$; i.e., the Larmor precession is fast compared to the delay between pulses 1 and 2 [29]. Here, $1 / \tau_{T}$ is the inverse spin lifetime of the trion, which is determined by the spin relaxation of the hole in the trion and its lifetime $1 / \tau_{T}=1 / T_{h}+1 / \tau_{r}$. Similar to the previous case, we have two terms in Eq. (4), corresponding to two different contributions. The fast decay is attributed to the trion lifetime [diagrams (d1) and (d2) in Fig. 1(d)], while the long-lived signal decays with $T_{2}^{e}$ [diagrams (d3) and (d4) in Fig. 1(d)], which corresponds to the electron spin dephasing in the ground state (transversal spin relaxation time). For $t_{2}=0$, the signal transforms into

$$
A_{\mathrm{PE}, \mathrm{t}_{2}=0}^{\perp} \propto e^{-\left[\left(2 t_{1}\right) / T_{2}\right]} \cos \left(\omega_{L} t_{1}\right) .
$$

Let us first consider the rephasing spectra for $t_{2}=0$,

$$
S_{I}\left(\Omega_{1}, \Omega_{3}\right)=\iint S_{I}\left(t_{1}, t_{3}\right) e^{i\left(\Omega_{1} t_{1}-\Omega_{3} t_{3}\right)} d t_{1} d t_{3},
$$

where $\Omega_{1}$ and $\Omega_{3}$ correspond to the absorption and emission optical frequencies. Fourier transformation of Eq. (1) with Eqs. (3) and (5) gives

$$
\begin{aligned}
S_{I}^{\|}\left(\Omega_{1}, \Omega_{3}\right) \propto \frac{\gamma e^{-\left\{\left[\sigma^{2}\left(\Omega_{1}-\bar{\Omega}_{0}\right)^{2}\right] / 2\right\}}}{4 \gamma^{2}+\left(\Omega_{1}-\Omega_{3}\right)^{2}}, \\
S_{I}^{\perp}\left(\Omega_{1}, \Omega_{3}\right)=S_{I}^{+}+S_{I}^{-}, \\
S_{I}^{ \pm}\left(\Omega_{1}, \Omega_{3}\right) \propto \frac{\gamma e^{-\left\{\left[\sigma^{2}\left(\Omega_{1}-\bar{\Omega}_{0}\right)^{2}\right] / 2\right\}}}{4 \gamma^{2}+\left(\Omega_{1}-\Omega_{3} \pm \omega_{L}\right)^{2}},
\end{aligned}
$$

where $\bar{\Omega}_{0}$ is the central frequency of the inhomogeneous ensemble with a half-width in frequency corresponding to $1 / \sigma$. Here, it is assumed that the inhomogeneous broadening $\Gamma=1 / \sigma$ is significantly larger than the homogeneous broadening $\gamma=1 / T_{2}$. This condition is fulfilled for the optical transitions to the localized $X^{-}$and $D^{0} X$ complexes [25,30-32]. The rephasing spectra for the co- and crosspolarized configurations are plotted in Figs. 2(a) and 2(b), respectively. The splitting of the diagonal line by the energy $\hbar \omega_{L}$ in the cross-polarized configuration clearly demonstrates the stimulated Raman process with an increase $\left(S_{I}^{+}\right)$ and a decrease $\left(S_{I}^{-}\right)$of the emission frequency compared to single excitation.

Particularly interesting are the Raman coherence spectra

$S_{I}\left(t_{1}, \Omega_{2}, \Omega_{3}\right)=\iint S_{I}\left(t_{1}, t_{2}, t_{3}\right) e^{-i\left(\Omega_{2} t_{2}+\Omega_{3} t_{3}\right)} d t_{2} d t_{3}$,

where $\Omega_{2}$ is the Raman coherence frequency.

For the copolarized configuration using Eqs. (1), (2), and (10), we obtain

$$
\begin{aligned}
S_{I}^{\|}\left(t_{1}, \Omega_{2}, \Omega_{3}\right) \propto & e^{-\left[\left(2 t_{1}\right) / T_{2}\right]} e^{-i \Omega_{3} t_{1}} \\
& \times e^{-\left\{\left[\sigma^{2}\left(\Omega_{3}-\bar{\Omega}_{0}\right)^{2}\right] / 2\right\}}\left(S_{I, T}^{\|}+S_{I, e}^{\|}\right), \\
S_{I, T}^{\|}= & 2 \cos ^{2}\left(\omega_{L} t_{1} / 2\right) \frac{\gamma_{r}}{\gamma_{r}^{2}+\Omega_{2}^{2}} \\
& +\sin ^{2}\left(\omega_{L} t_{1} / 2\right) \frac{\gamma_{T}}{\gamma_{T}^{2}+\Omega_{2}^{2}}, \\
S_{I, e}^{\|}= & \sin ^{2}\left(\omega_{L} t_{1} / 2\right) \frac{\gamma_{1, e}}{\gamma_{1, e}^{2}+\Omega_{2}^{2}},
\end{aligned}
$$

where $\gamma_{r}=1 / \tau_{r}, \gamma_{T}=1 / \tau_{T}$, and $\gamma_{1, e}=1 / T_{1}^{e}$. The corresponding 2DFTS image is shown in Fig. 3(a) for the case when $\gamma_{r}=\gamma_{T} \gg \gamma_{1, e}$. In this case, two Lorentzian peaks are 

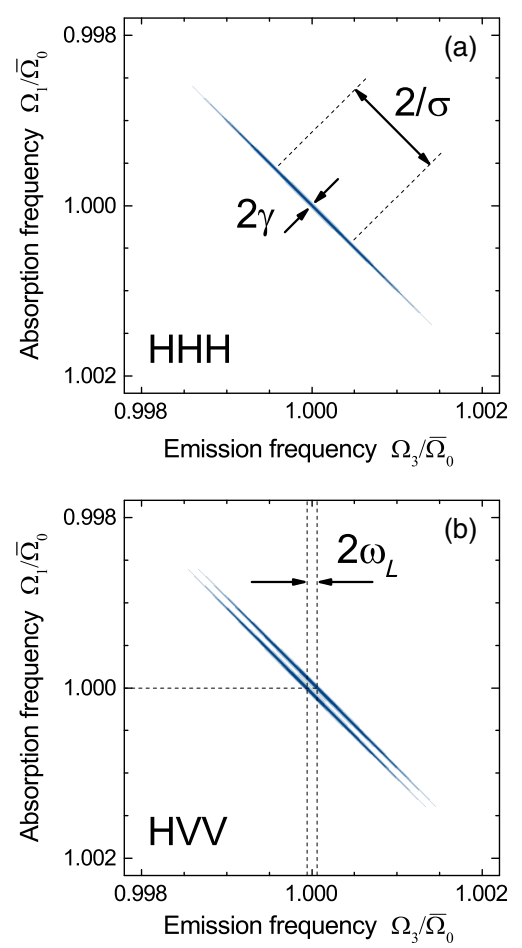

FIG. 2. Contour plots of the 2D Fourier rephasing spectra $S_{I}\left(\Omega_{1}, \Omega_{3}\right)$ in the copolarized HHH (a) and in cross-polarized HVV (b) polarization configurations after Eqs. (7) and (8), respectively. The following parameters are used: $\hbar \bar{\Omega}_{0}=1.6 \mathrm{eV}$, $\hbar \Gamma=\hbar / \sigma=1 \mathrm{meV}, \hbar \gamma=\hbar / T_{2}=10 \mu \mathrm{eV}$, and $\hbar \omega_{L}=100 \mu \mathrm{eV}$.

centered at $\Omega_{2}=0$ [see the cross section at $\Omega_{3}=\bar{\Omega}_{0}$ in Fig. 3(c)]. Their widths are given by $\gamma_{r}$ and $\gamma_{1, e}$, and their relative amplitudes depend on $\omega_{L} t_{1}$. The spectrum can be used to evaluate the lifetimes of the excited states $\left(\gamma_{r}\right)$ and the time of population relaxation between the ground states $|1\rangle$ and $|2\rangle\left(\gamma_{1, e}\right)$.

For the cross-polarized configuration using Eqs. (1), (4), and (10), we obtain

$$
\begin{aligned}
S_{I}^{\perp}\left(t_{1}, \Omega_{2}, \Omega_{3}\right) \propto & e^{-\left[\left(2 t_{1}\right) / T_{2}\right]} e^{-i \Omega_{3} t_{1}} \\
& \times e^{-\left\{\left[\sigma^{2}\left(\Omega_{3}-\bar{\Omega}_{0}\right)^{2}\right] / 2\right\}}\left(S_{I, T}^{\perp}+S_{I, e}^{\perp}\right), \\
S_{I, T}^{\perp}= & 2 \cos \left(\omega_{L} t_{1}\right) \frac{\gamma_{T}}{\gamma_{T}^{2}+\Omega_{2}^{2}}, \\
S_{I, e}^{\perp}= & \frac{\gamma_{2, e} e^{i \omega_{L} t_{1}}}{\gamma_{2, e}^{2}+\left(\Omega_{2}+\omega_{L}\right)^{2}}+\frac{\gamma_{2, e} e^{-i \omega_{L} t_{1}}}{\gamma_{2, e}^{2}+\left(\Omega_{2}-\omega_{L}\right)^{2}},
\end{aligned}
$$

where $\gamma_{2, e}=1 / T_{2}^{e}$. This 2DFTS spectrum is shown in Fig. 3(b). It is worth mentioning that in the HVV configuration, only the imaginary part is present for $\omega_{L} t_{1}=\pi / 2$. It contains two peaks with different signs (dispersive shape) at frequencies $\Omega_{2}= \pm \omega_{L}$ [see Fig. 3(d)]. The widths of these peaks are given by $\gamma_{2, e}$.
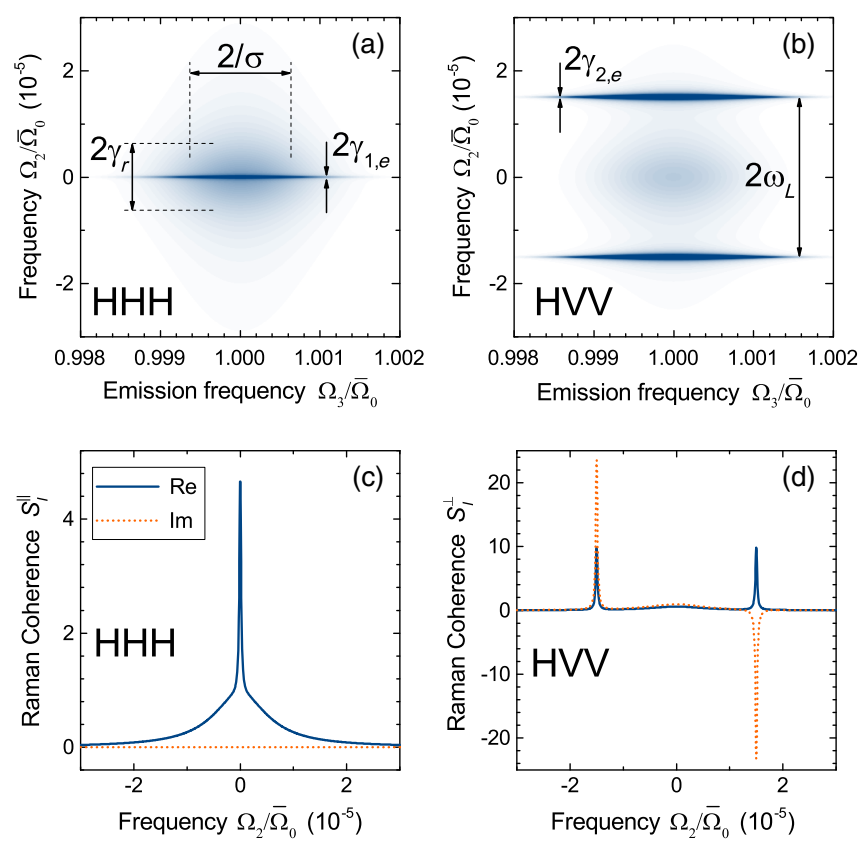

FIG. 3. Contour plots of the absolute value of the Raman coherence 2D Fourier spectra in the copolarized HHH (a) and cross- polarized HVV (b) polarization configuration and their cross sections at the optical frequency $\Omega_{3}=\bar{\Omega}_{0}$ (c,d), respectively. The following parameters are used: $\hbar \bar{\Omega}_{0}=1.6 \mathrm{eV}$, $\hbar \Gamma=\hbar / \sigma=1 \mathrm{meV}, \hbar \gamma_{r}=\hbar \gamma_{T}=10 \mu \mathrm{eV}, \hbar \gamma_{1, e}=\hbar \gamma_{2, e}=0.3 \mu \mathrm{eV}$, $\hbar \omega_{L}=24 \mu \mathrm{eV}$, and $t_{1}=26.7 \mathrm{ps}$.

Thus, the 2DFTS spectra measured in the cross-polarized configuration allow us to evaluate the coherence times and the energy splitting between the ground-state levels $\hbar \omega_{L}$. The measurement of the splitting works even if it significantly undercuts the homogeneous spectral width of the optical transitions $(\gamma)$. Thus, this method can be used for high-resolution spectroscopy of the ground state. The advantage is the possibility to determine the splitting of the ground states for excitation at a particular photon energy $\omega=\Omega_{1}$.

An excellent example and demonstration of this powerful technique is the determination of the spin splittings of different complexes that exist simultaneously in the very same sample, e.g., $X^{-}$and $D^{0} X$, as shown in the next section. This information cannot be obtained using pure spin resonance techniques where the optical excitation with $\Omega_{1}$ is absent. Therefore, in this particular case, we perform optically detected magnetic resonance using coherent optical spectroscopy. Eventually, the optical coherence initiated by the laser pulse plays an essential role during the excitation and the final emission process at $\Omega_{1}$ and $\Omega_{3}$ optical frequency, respectively. Otherwise, the Raman coherence cannot be restored, which is in contrast to conventional time-resolved pump-probe Faraday rotation measurements [33-35]. 


\section{OPTICALLY DETECTED COHERENT SPECTROSCOPY OF CdTe QW WITH RESIDENT ELECTRONS}

The investigated sample comprises a 20-nm-thick CdTe QW sandwiched between $\mathrm{Cd}_{0.76} \mathrm{Mg}_{0.24} \mathrm{Te}$ barriers. The QW was grown by molecular-beam epitaxy on a (100)GaAs substrate, onto which a thick $(\mathrm{Cd}, \mathrm{Mg})$ Te buffer was deposited followed by a short-period superlattice and a 100-nm $\mathrm{Cd}_{0.76} \mathrm{Mg}_{0.24} \mathrm{Te}$ barrier. The structure was not intentionally doped with donors; however, the unavoidable background of impurities results in localized resident carriers that originate from the barriers as well as from electrons bound to donors in the QW. The density of these electrons, $n_{e} \leq 10^{10} \mathrm{~cm}^{-2}$, in the QW is low so that the exciton Bohr radius $a_{B} \ll 1 / \sqrt{n_{e}}$, which allows us to consider each resident electron as isolated and noninteracting with other electrons. The photoluminescence (PL) spectrum is shown in Fig. 4(b) and consists of several spectral lines, which we attribute to the exciton $(X)$, the trion $\left(X^{-}\right)$, and the donor-bound exciton $\left(D^{0} X\right)$. In the experiments, the sample is mounted in the Voigt geometry in a liquid-helium split-coil magnetocryostat and is kept at a temperature of $T=2 \mathrm{~K}$. The direction of the magnetic field is parallel to the quantum-well plane $(\mathbf{B} \| x)$. The applied magnetic field $B=260 \mathrm{mT}$ results in an energy splitting of $\hbar \omega_{L}=24 \mu \mathrm{eV}$, which is small compared to the thermal energy $k_{B} T=170 \mu \mathrm{eV}$.

(a)

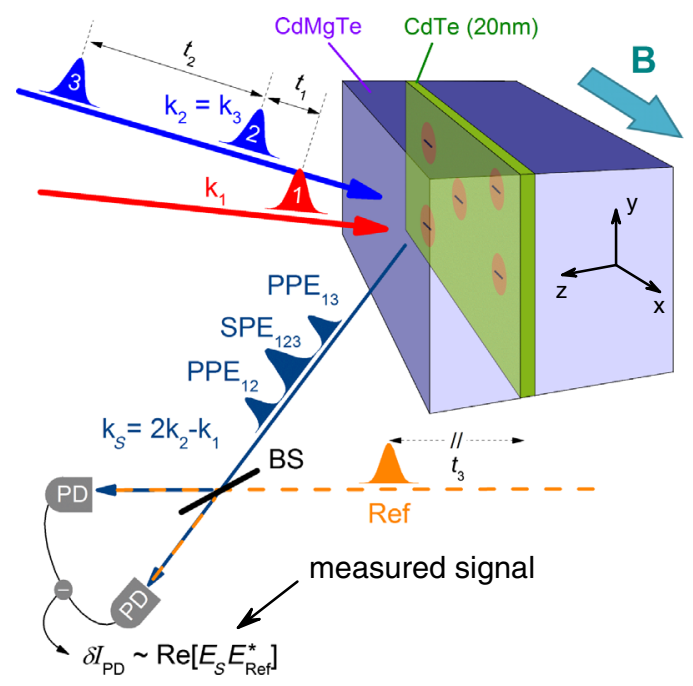

For the photon-echo experiments, we use a sequence of three excitation pulses with variable delays $t_{1}$ between pulses 1 and 2 and $t_{2}$ between pulses 2 and 3 [see Fig. 4(a)]. All pulses are obtained by splitting the emission of a tunable self-mode-locked Ti-Sa oscillator with a repetition frequency of $75.75 \mathrm{MHz}$. The duration of the pulses is $2 \mathrm{ps,}$ and their spectral width is $\hbar \delta \omega=0.9 \mathrm{meV}$. An example of the laser spectrum is shown in Fig. 4(b). These spectrally narrow optical pulses allow us to achieve a high selectivity for excitation of the exciton complexes, e.g., $X, X^{-}$or $D^{0} X$. In addition, they also prevent excitation of higher-energy states, e.g., light-hole transitions. The delay between the pulses is controlled using $27-\mathrm{cm}$-long motorized translation stages by which we can cover maximum delays of about $1.8 \mathrm{~ns}$. The optical pulse 1 hits the sample at an incidence angle of $6^{\circ}$. The pulses 2 and 3 both hit at a $7^{\circ}$ angle, so their wave vectors are equal $\left(\mathbf{k}_{2}=\mathbf{k}_{3}\right)$. The beams are focused onto the sample at a spot with a diameter of about $200 \mu \mathrm{m}$. The energy density of each pulse is kept below $30 \mathrm{~nJ} / \mathrm{cm}^{2}$ in order to remain in the $\chi^{(3)}$ regime; i.e., the photon-echo intensity depends linearly on the intensity of each of the beams. The polarization of the excitation pulses is controlled with retardation plates in conjunction with polarizers. The FWM signal is collected along the $\mathbf{k}_{S}=2 \mathbf{k}_{2}-\mathbf{k}_{1}$ direction in reflection geometry. Here, the phase matching condition is not sensitive to the $z$ component of the wave vector $\mathbf{k}_{S}$ since the signal originates from

(b)
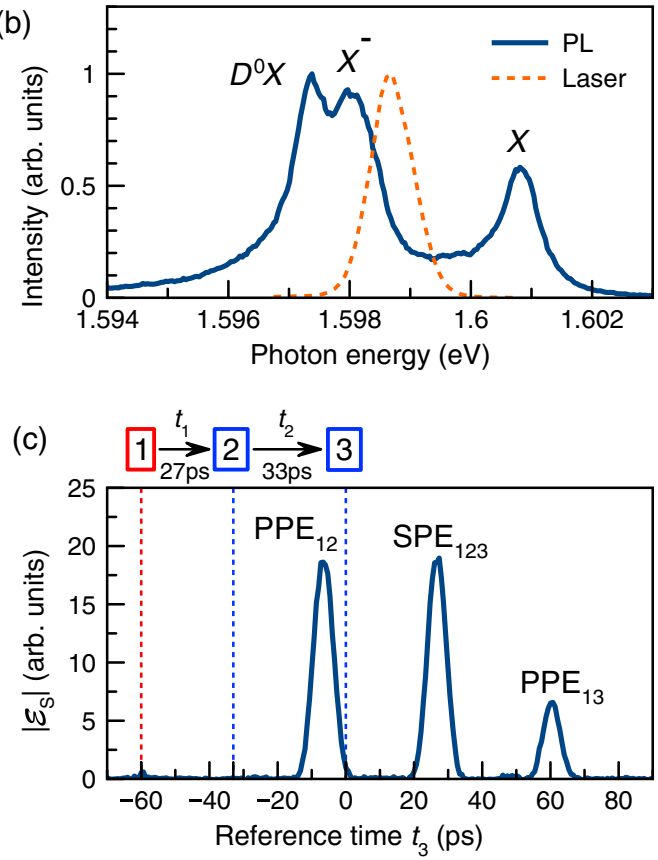

FIG. 4. (a) Schematic illustration of the heterodyne-detected photon echoes in reflection geometry. BS and PD denote the beam splitter and photodiode, respectively. (b) PL spectrum (the solid line) of the investigated 20-nm-thick $\mathrm{CdTe} /(\mathrm{Cd}, \mathrm{Mg}) \mathrm{Te} \mathrm{QW}$ structure measured at temperature $T=2 \mathrm{~K}$ for above-barrier excitation with photon energy $2.33 \mathrm{eV}$. The laser spectrum is shown by the dashed line. (c) Time-resolved cross correlation of the resulting FWM signal $\left|\mathcal{E}_{S}\left(t_{3}\right)\right|$ measured for $t_{1}=27 \mathrm{ps}$ and $t_{2}=33$ ps as well as $\hbar \omega=1.597 \mathrm{eV}$. The signal is given by photon echoes involving different pulse sequences: $\mathrm{PPE}_{12}$ and $\mathrm{PPE}_{13}$ correspond to the two-pulse sequences 1-2 and 1-3, respectively. $\mathrm{SPE}_{123}$ corresponds to the three-pulse sequence 1-2-3. 
the QW layer, which has a thickness that is significantly smaller than the wavelength of light in CdTe.

In order to resolve the transient profile of the coherent optical response $E_{S}(t)$, we use interferometric heterodyne detection [36,37], where the FWM signal and the reference beams are overlapped at the balanced detector [see Fig. 4(a)]. The reference pulse with optical field $E_{\text {Ref }}$ is obtained from the same laser oscillator, and its delay can be varied with a separate translation stage. The optical frequencies of pulse 1 and the reference pulse are shifted by $+40 \mathrm{MHz}$ and $-41 \mathrm{MHz}$ with acousto-optical modulators. The resulting interference signal $\operatorname{Re}\left[E_{S} E_{\mathrm{Ref}}^{*}\right]$ at the photodiode is detected with a high-frequency lock-in amplifier at $\left|2 \omega_{2}-\omega_{1}-\omega_{\operatorname{Ref}}\right|=1 \mathrm{MHz}$. The phase of this signal is locked at short times because all of the pulses originate from the same laser source. However, random fluctuations of the optical phase in the different beam paths at time scales longer than $1 \mathrm{~ms}$ are not suppressed because no active stabilization of beam paths is implemented in our experiment. Therefore, only the amplitude of the signal is accessible in the measurement procedure. Still, heterodyne detection provides a high-sensitivity, background-free measurement of the crosscorrelation function for the absolute value of the FWM electric field amplitude $\left|\mathcal{E}_{S}\left(t_{3}\right)\right| \propto\left|\int E_{S}(t) E_{\text {Ref }}^{*}\left(t-t_{3}\right) d t\right|$ when scanning the reference pulse delay time $t_{3}$, which is taken to be relative to the arrival time of pulse 3 [see Figs. 1 and 4(a)].

Figure 4(c) shows a typical time-resolved FWM signal $\left|\mathcal{E}_{S}\left(t_{3}\right)\right|$ measured for $t_{1}=27 \mathrm{ps}$ and $t_{2}=33 \mathrm{ps}$ with excitation at photon energy $\hbar \omega=1.597 \mathrm{eV}$. The coherent optical response is fully given by photon echoes. Because $\mathbf{k}_{2}=\mathbf{k}_{3}$, several echoes are emitted along the phasematching direction $\mathbf{k}_{S}$. Two that appear at $t_{3}=t_{1} \pm t_{2}$ correspond to primary photon echoes (PPE), which result from the two-pulse sequences 1-2 and 1-3 and are labeled correspondingly as $\mathrm{PPE}_{12}$ and $\mathrm{PPE}_{13}$ in Fig. 4(c). The peak located at $t_{3}=t_{1}$ corresponds to the stimulated photon echo (SPE), which is induced by the three-pulse sequence 1-2-3. Thus, the use of heterodyne detection allows us to distinguish between different echoes and to record the time evolution of the PE $A_{\mathrm{PPE}}$ and SPE $A_{\mathrm{SPE}}$ peak amplitudes by choosing the proper detection delay time $t_{3}$.

Figure 5 summarizes the spectral dependence of the coherent optical response measured in the copolarized configuration at $B=0$. The peak amplitudes $A_{\mathrm{PPE}}$ and $A_{\mathrm{SPE}}$ as a function of $2 t_{1}$ and $t_{2}$ for different $\hbar \omega$ are shown in Figs. 5(a) and 5(b), respectively. From the exponential decay of the peak amplitudes, we evaluate the coherence time $T_{2}$ and the lifetime $T_{1}$ of the photoexcited complexes following Eqs. (2) and (3). A proper choice of the excitation photon energy $\hbar \omega$ enables us to determine the homogeneous linewidth $\gamma$ and the population decay rate $\gamma_{r}=$ $1 / T_{1}$ of the particular optical transition, e.g., $X, X^{-}$or $D^{0} X$. The spectral dependencies of the PPE signal strength $A_{\mathrm{PPE}}^{0}$, $\gamma$, and $\gamma_{r}$ are plotted in Fig. 5(c).
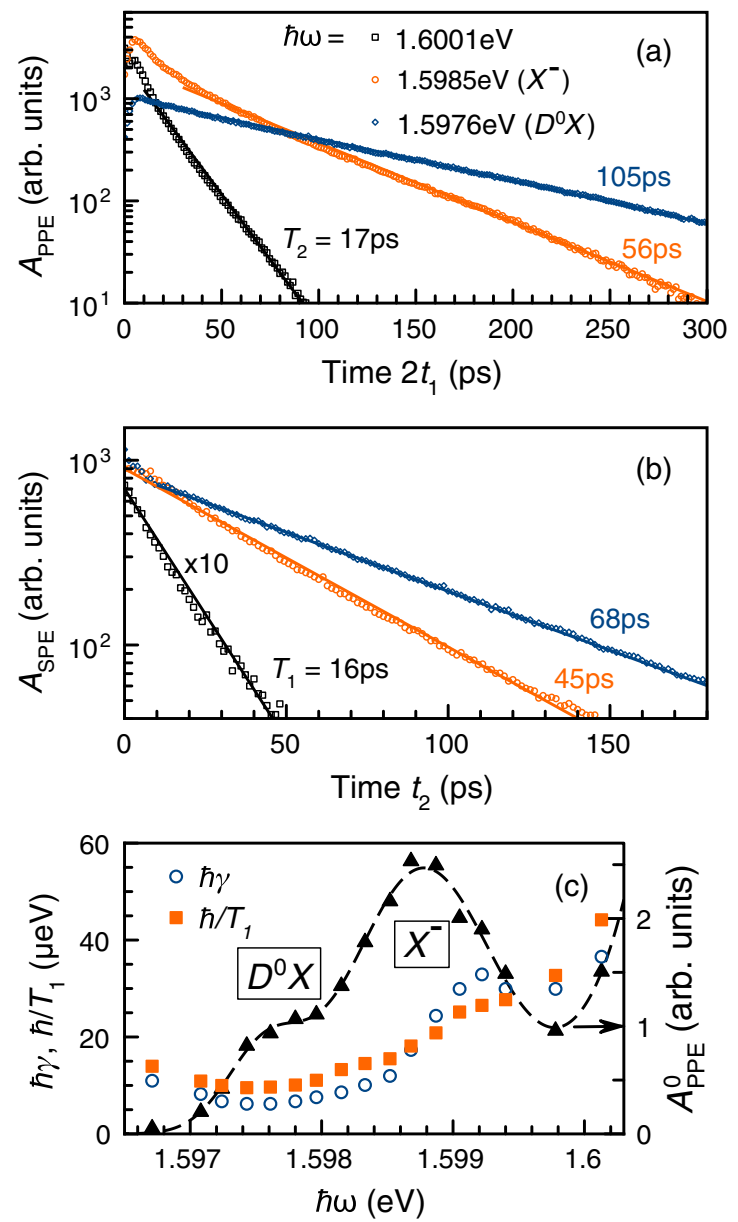

FIG. 5. Spectral dependence of the $A_{\mathrm{PPE}}$ and $A_{\mathrm{SPE}}$ transients at $B=0$. (a,b) $A_{\mathrm{PPE}}$ and $A_{\mathrm{SPE}}$ measured as a function of $2 t_{1}$ and $t_{2}$, respectively. The SPE is measured for $t_{1}=27 \mathrm{ps}$. (c) Spectral dependence of $A_{\mathrm{PPE}}^{0}=A_{\mathrm{PPE}}\left(t_{1}=0\right), \hbar \gamma$ and $\hbar / T_{1}$, evaluated from the exponential fits to the echo transients for different photon energies $\hbar \omega$ [see the solid lines in (a) and (b)].

For resonant excitation of the low energy tail of the localized excitons at $\hbar \omega=1.601 \mathrm{eV}$, we obtain short decoherence times limited to $17 \mathrm{ps}$, i.e., $\hbar \gamma=38 \mu \mathrm{eV}$. For higher $\hbar \omega$ corresponding to excitation of free excitons $(X)$, the linewidth becomes even larger, reaching values of $150 \mu \mathrm{eV}[25,27]$. For the trions and donor-bound excitons, the homogeneous linewidths are significantly narrower because of the stronger localization of these complexes. Here, we obtain $\hbar \gamma \approx 12 \mu \mathrm{eV}$ for $X^{-}$and $\hbar \gamma \approx 6 \mu \mathrm{eV}$ for $D^{0} X$. At lower energies, $\hbar \omega \leq 1.598 \mathrm{eV}$, we observe that the linewidth is determined mainly by the lifetime, i.e., $T_{2} \sim 2 T_{1}$ and thus, the pure dephasing is weak. Note that the intentionally chosen spectrally narrow laser pulses $(\hbar \delta \omega=0.9 \mathrm{meV}$ ) also help to suppress many-body interactions between different photoexcited complexes. For example, using spectrally broad femtosecond pulses, one would simultaneously excite excitons and trions. As a result, the exciton-trion interaction would lead to a significant increase of the homogeneous linewidth for the trion 
transition, which would become comparable to the one measured for the $X$ [25].

In order to evaluate the Zeeman energy splitting of the ground electronic states, we concentrate on the $A_{\mathrm{SPE}}$ transients, setting the reference pulse delay $t_{3}=t_{1}$ and performing scans as a function of $t_{2}$ for different excitation photon energies $\hbar \omega$ in the cross-polarized configuration (HVV). The data are summarized in Fig. 6. Contour plots of the SPE peak amplitude as a function of $t_{2}$ and $\hbar \omega$ measured at $B=260 \mathrm{mT}$ for $t_{1}=27 \mathrm{ps}$ are shown in Fig. 6(a). Exemplary curves taken at $\hbar \omega=1.5972 \mathrm{eV}$ and $1.5985 \mathrm{eV}$, corresponding to excitation of the $D^{0} X$ and $X^{-}$ optical transitions, respectively, are shown in Fig. 6(b). When $t_{2}$ is varied, we observe an oscillatory signal that decays on a long time scale of several ns. Note that the long-lived signal is present only in the spectral region $1.597-1.599 \mathrm{eV}$, where the $X^{-}$and the $D^{0} X$ resonances are located; i.e., it is necessary to address resident carriers for storing optical information on such long time scales.

Using Eq. (4), we evaluate the spectral dependence of the long-lived SPE signal strength $A_{\mathrm{SPE}}^{e, 0}$, the absolute value of the electron $g$ factor $|g|=\hbar \omega_{L} / \mu_{B} B$, and the decay rate $\gamma_{2, e}$, which are plotted in Fig. 6(c). In accordance with Eq. (12), these parameters allow us to restore
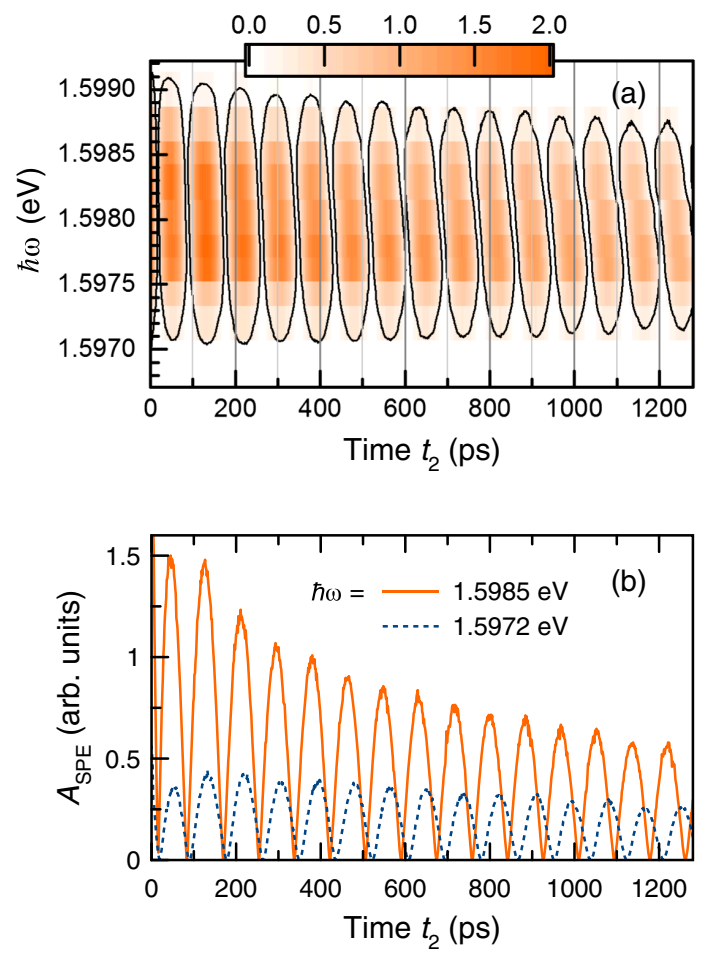

the 2DFTS Raman coherence image $\left|S_{I}^{\perp}\left(\Omega_{2}, \Omega_{3}\right)\right| \approx$ $\sum A_{\mathrm{SPE}}^{e, 0}\left|S_{I, e}^{\perp}\left(\Omega_{2}\right)\right| \exp \left\{-\left[\sigma^{2}\left(\Omega_{3}-\omega\right)^{2}\right] / 2\right\}$, where we set $2 / \sigma=\delta \omega$ and neglect the $S_{I, T}^{\perp}$ term since $\gamma_{T} \gg \gamma_{2, e}$. The sum is taken over all excitation energies $\hbar \omega$ used in the experiment. The resulting contour plot is shown in Fig. 6(d). Note that we cannot distinguish between real and imaginary contributions to the 2DFTS signal. However, as follows from the theoretical description in Sec. II, this is not crucial for the evaluation of the energy splitting in the ground state in the case of isolated localized electrons. Nevertheless, phase-stabilized measurements have great potential for in-depth studies of many-body interactions in an ensemble of resident electrons similar to that obtained for photoexcited exciton complexes [10].

The most striking feature of Fig. 6(d) is the variation of the Raman coherence peak with photon energy $\hbar \Omega_{3}$ demonstrating a steplike behavior and clearly showing that $\hbar \omega_{L}$ increases from 24.0 to $24.6 \mu \mathrm{eV}$ when the excitation energy is varied from $D^{0} X$ to $X^{-}$. The extracted $g$ factors are $|g|=1.595$ and 1.635 for the resident electrons bound to a donor and localized in a potential fluctuation, respectively [see also Fig. 6(c)]. The small difference between these values has the opposite trend to that expected for free electrons in CdTe/(Cd, Mg)Te QWs and (Cd,Mg)Te [38].
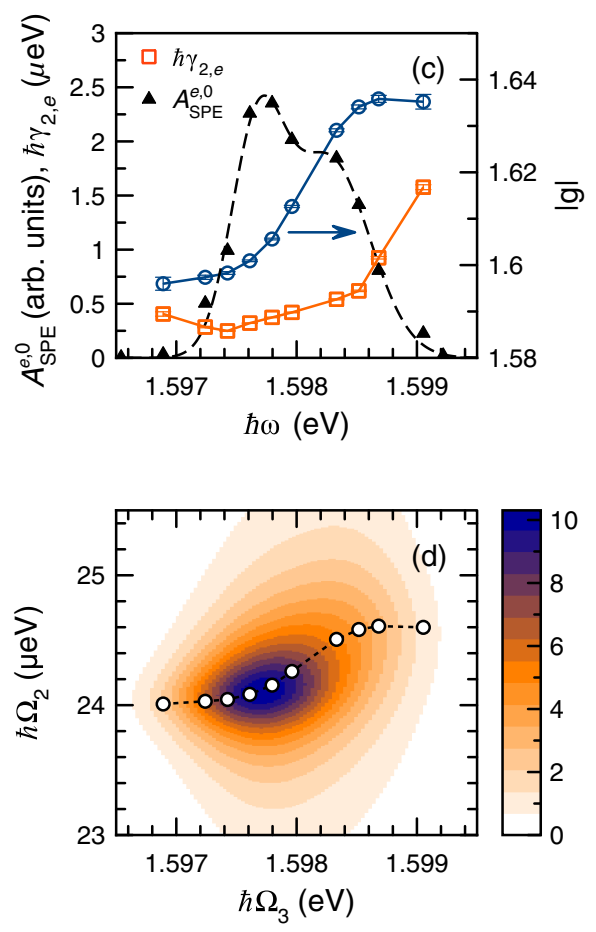

FIG. 6. (a) Contour plot of the long-lived SPE measured in the HVV polarization configuration as a function of the excitation photon energy $\hbar \omega$ and delay time $t_{2}$. The data are taken at $B=260 \mathrm{mT}, t_{1}=27 \mathrm{ps}$. The solid black lines indicate the signal level with $10 \%$ of the maximum intensity. (b) Exemplary transients for given photon energies of $1.5972 \mathrm{eV}$ and $1.5985 \mathrm{eV}$ corresponding to optical excitation of the $D^{0} X$ and $X^{-}$complexes, respectively. (c) Spectral dependence of the long-lived SPE signal strength $A_{\mathrm{SPE}}^{e, 0}=$ $A_{\mathrm{SPE}}^{e}\left(t_{2}=0\right)$ (left axis), the linewidth $\hbar \gamma_{2, e}$ (left axis), and the electron $g$ factor (right axis) evaluated from the $A_{\mathrm{SPE}}\left(t_{2}\right)$ transients at $B=260 \mathrm{mT}$. (d) The 2DFTS Raman coherence image $\left|S_{I}^{\perp}\left(\Omega_{2}, \Omega_{3}\right)\right|$ reconstructed from (c). The spectral dependence of $\hbar \omega_{L}$ is shown by the dots. 
The electron $g$ factor is controlled by the admixture of valence-band states to the conduction band, which in turn is dependent not only on the band gap energy but also on the electron and hole localization. This localization is different for the $D^{0} X$ and $X^{-}$complexes, which results in the measured differences of the $g$ values.

Finally, we discuss the spectral dependence of the decay rate $\gamma_{2, e}$, which increases from $0.25 \mu \mathrm{eV}$ for donor-bound electrons to $1.5 \mu \mathrm{eV}$ for high-energy trions. There are several mechanisms that can contribute to the decay of the long-lived signal. For localized noninteracting electrons, spin relaxation and hopping between localization sites are relevant. The latter mechanism deserves special attention as it may be spin conserving and, therefore, does not give a contribution to conventional pump-probe measurements where the decay of the signal is determined solely by the spin relaxation. The increase of $\gamma_{2, e}$ with increasing $\hbar \omega$ indicates that hopping of electrons between localization sites plays an important role for states with weaker localization. Simultaneously, for the donor-bound electrons with strongest localization, the signal decay approaches the values measured on similar structures using the pump-probe technique (about 3-10 ns) [34].

Thus, our results demonstrate that the decay of Raman coherence in an electron spin ensemble measured by photon echoes provides access to the local relaxation processes, such as hopping of carriers between localization sites or spin interactions between electrons within the ensemble (e.g., spin flip-flops). This is because every individual electron in the ground state contributes to the coherent optical response only if it is addressed by all three optical pulses sequentially. Moreover, in photon-echo experiments, because of dephasing of optically excited states between the first and second optical pulses, no macroscopic spin polarization is created in the ground state after the stimulated Raman process. In contrast, timeresolved pump-probe Faraday rotation [33-35] and transient spin-grating techniques [39-41] detect the evolution of the macroscopic spin polarization for a large electron ensemble, and local relaxation processes cannot be probed directly. Therefore, a comparison of the signal decays recorded with different experimental techniques can be used to obtain the full and self-consistent physical picture of the spin dynamics in electron ensembles.

\section{CONCLUSIONS}

In conclusion, we have demonstrated that twodimensional Fourier spectroscopy addressing photon echoes can be successfully applied for the evaluation of tiny splittings between ground-state energy levels which are optically coupled to a common excited state in, e.g., a $\Lambda$-type scheme. We have shown that the stimulated steplike Raman process induced by the sequence of two pulses creates a coherent superposition of the ground-state doublet which can be retrieved only by optical means because of selective excitation of the same spin subensemble with the third pulse. This provides the unique opportunity to distinguish between several electron spin species in a large ensemble of emitters. As a proof of principle, we have applied this method to an $n$-type $\mathrm{CdTe} /(\mathrm{Cd}, \mathrm{Mg}) \mathrm{Te}$ quantum-well system for which the Zeeman splitting difference in the sub- $\mu \mathrm{eV}$ range between donor-bound electrons and electrons localized on potential fluctuations has been resolved, while the homogeneous linewidth of the optical transitions is 2 orders of magnitude larger than this splitting. Our results pave the way for further developing two-dimensional Fourier imaging into a high-resolution spectroscopy tool, independent of the nature of the energy splitting in the ground state.

\section{ACKNOWLEDGMENTS}

We acknowledge financial support from the Deutsche Forschungsgemeinschaft (DFG) through the Collaborative Research Centre TRR 142 (Project No. A02) and the International Collaborative Research Centre TRR 160, the latter of which is also supported by the Russian Foundation of Basic Research (Project No. 15-52-12016 NNIO_a). The research in Poland was partially supported by the National Science Centre (Poland) through Grants No. DEC-2012/06/A/ST3/00247 and No. DEC- 2014/14/ M/ST3/00484, as well as by the Foundation for Polish Science through the IRA Programme co-financed by EU within SG OP.

[1] W. Demtröder, Laser Spectroscopy 2 (Springer, Berlin, 2015), Chap. 7.

[2] S. T. Cundiff and S. Mukamel, Optical Multidimensional Coherent Spectroscopy, Phys. Today 66, No. 7, 44 (2013).

[3] M. Fleischhauer, A. Imamoglu, and J. P. Marangos, Electromagnetically Induced Transparency: Optics in Coherent Media, Rev. Mod. Phys. 77, 633 (2005).

[4] J. Shah, Ultrafast Spectroscopy of Semiconductors and Semiconductor Nanostructures, Springer Series in SolidState Sciences (Springer Science \& Business Media, New York, 1999), Vol. 115.

[5] S. Mukamel, Principles of Nonlinear Optical Spectroscopy (Oxford University, New York, 1995).

[6] T. Zhang, I. Kuznetsova, T. Meier, X. Li, R. P. Mirin, P. Thomas, and S. T. Cundiff, Polarization-Dependent Optical $2 D$ Fourier Transform Spectroscopy of Semiconductors, Proc. Natl. Acad. Sci. U.S.A. 104, 14227 (2007).

[7] L. Yang, I. V. Schweigert, S. T. Cundiff, and S. Mukamel, Two-Dimensional Optical Spectroscopy of Excitons in Semiconductor Quantum Wells: Liouville-Space Pathway Analysis, Phys. Rev. B 75, 125302 (2007).

[8] K. W. Stone, K. Gundogdu, D. B. Turner, X. Li, S. T. Cundiff, and K. A. Nelson, Two-Quantum 2D FT Electronic Spectroscopy of Biexcitons in GaAs Quantum Wells, Science 324, 1169 (2009). 
[9] J. Kasprzak, B. Patton, V. Savona, and W. Langbein, Coherent Coupling Between Distant Excitons Revealed by Two-Dimensional Nonlinear Hyperspectral Imaging, Nat. Photonics 5, 57 (2011).

[10] S. Cundiff, T. Zhang, A. Bristow, D. Karaiskaj, and D. Xinccan, Optical Two-Dimensional Fourier Transform Spectroscopy of Semiconductor Quantum Wells, Acc. Chem. Res. 42, 1423 (2009).

[11] M. O. Scully and M. S. Zubairy, Quantum Optics (Cambridge University Press, Cambridge, England, 1997), Chap. 7.

[12] K. Hammerer, A. S. Sørensen, and E. S. Polzik, Quantum Interface Between Light and Atomic Ensembles, Rev. Mod. Phys. 82, 1041 (2010).

[13] Spin Physics in Semiconductors, edited by M. Dyakonov (Springer-Verlag, Berlin, 2008).

[14] K.-M. C. Fu, C. Santori, C. Stanley, M. C. Holland, and Y. Yamamoto, Coherent Population Trapping of Electron Spins in a High-Purity n-Type GaAs Semiconductor, Phys. Rev. Lett. 95, 187405 (2005).

[15] M. Sladkov, A. U. Chaubal, M. P. Bakker, A. R. Onur, D. Reuter, A. D. Wieck, and C. H. van der Wal, Electromagnetically Induced Transparency with an Ensemble of Donor-Bound Electron Spins in a Semiconductor, Phys. Rev. B 82, 121308 (2010).

[16] H. Wang and S. O'Leary, Electromagnetically Induced Transparency from Electron Spin Coherences in Semiconductor Quantum Wells, J. Opt. Soc. Am. B 29, A6 (2012).

[17] X. Xu, B. Sun, P. R. Berman, D. G. Steel, A. S. Bracker, D. Gammon, and L. J. Sham, Coherent Population Trapping of an Electron Spin in a Single Negatively Charged Quantum Dot, Nat. Phys. 4, 692 (2008).

[18] D. Brunner, B. D. Gerardot, P. A. Dalgarno, G. Wüst, K. Karrai, N. G. Stoltz, P. M. Petroff, and R. J. Warburton, A Coherent Single-Hole Spin in a Semiconductor, Science 325, 70 (2009).

[19] J. Hansom, C. H. H. Schulte, C. Le Gall, C. Matthiesen, E. Clarke, M. Hugues, J. M. Taylor, and M. Atatüre, Environment-Assisted Quantum Control of a Solid-State Spin via Coherent Dark States, Nat. Phys. 10, 725 (2014).

[20] A. Greilich, D. R. Yakovlev, A. Shabaev, Al. L. Efros, I. A. Yugova, R. Oulton, V. Stavarache, D. Reuter, A. Wieck, and M. Bayer, Mode Locking of Electron Spin Coherences in Singly Charged Quantum Dots, Science 313, 341 (2006).

[21] S. G. Carter, Z. Chen, and S. T. Cundiff, Ultrafast BelowResonance Raman Rotation of Electron Spins in GaAs Quantum Wells, Phys. Rev. B 76, 201308 (2007).

[22] D. Press, T. D. Ladd, B. Zhang, and Y. Yamamoto, Complete Quantum Control of a Single Quantum Dot Spin Using Ultrafast Optical Pulses, Nature (London) 456, 218 (2008).

[23] I. Broser, B. Lummer, R. Heitz, and A. Hoffmann, Degenerate Four-Wave Mixing at Bound Excitons in II-VI Semiconductors, J. Cryst. Growth 138, 809 (1994).

[24] D. Brinkmann, J. Kudrna, P. Gilliot, B. Hönerlage, A. Arnoult, J. Cibert, and S. Tatarenko, Trion and Exciton Dephasing Measurements in Modulation-Doped Quantum Wells: A Probe for Trion and Carrier Localization, Phys. Rev. B 60, 4474 (1999).

[25] G. Moody, I. A. Akimov, H. Li, R. Singh, D. R. Yakovlev, G. Karczewski, M. Wiater, T. Wojtowicz, M. Bayer, and
S. T. Cundiff, Coherent Coupling of Excitons and Trions in a Photoexcited CdTe/CdMgTe Quantum Well, Phys. Rev. Lett. 112, 097401 (2014).

[26] F. Fras, Q. Mermillod, G. Nogues, C. Hoarau, C. Schneider, M. Kamp, S. Höfling, W. Langbein, and J. Kasprzak, MultiWave Coherent Control of a Solid-State Single Emitter, Nat. Photonics 10, 155 (2016).

[27] L. Langer, S. V. Poltavtsev, I. A. Yugova, D. R. Yakovlev, G. Karczewski, T. Wojtowicz, J. Kossut, I. A. Akimov, and M. Bayer, Magnetic-Field Control of Photon Echo from the Electron-Trion System in a CdTe Quantum Well: Shuffling Coherence Between Optically Accessible and Inaccessible States, Phys. Rev. Lett. 109, 157403 (2012).

[28] L. Langer, S. V. Poltavtsev, I. A. Yugova, M. Salewski, D. R. Yakovlev, G. Karczewski, T. Wojtowicz, I. A. Akimov, and M. Bayer, Access to Long-Term Optical Memories Using Photon Echoes Retrieved from Semiconductor Spins, Nat. Photonics 8, 851 (2014).

[29] See Supplemental Material at http://link.aps.org/ supplemental/10.1103/PhysRevX.7.031030: Sec. I gives the theoretical description of three-pulse echo for different polarization configurations; Sec. II gives double-sided Feynman diagrams.

[30] G. Noll, U. Siegner, S. G. Shevel, and E. O. Göbel, Picosecond Stimulated Photon Echo Due to Intrinsic Excitations in Semiconductor Mixed Crystals, Phys. Rev. Lett. 64, 792 (1990).

[31] M. Koch, D. Weber, J. Feldmann, E. O. Göbel, T. Meier, A. Schulze, P. Thomas, S. Schmitt-Rink, and K. Ploog, Subpicosecond Photon-Echo Spectroscopy on GaAs/AlAs Short-Period Superlattices, Phys. Rev. B 47, 1532 (1993).

[32] S. V. Poltavtsev, A. N. Kosarev, I. A. Akimov, D. R. Yakovlev, S. Sadofev, J. Puls, S. P. Hoffmann, M. Albert, C. Meier, T. Meier, and M. Bayer, Time-Resolved Photon Echoes from Donor-Bound Excitons in ZnO Epitaxial Layers, Phys. Rev. B 96, 035203 (2017).

[33] S. A. Crooker, D. D. Awschalom, J. J. Baumberg, F. Flack, and N. Samarth, Optical Spin Resonance and Transverse Spin Relaxation in Magnetic Semiconductor Quantum Wells, Phys. Rev. B 56, 7574 (1997).

[34] E. A. Zhukov, D. R. Yakovlev, M. Bayer, M. M. Glazov, E. L. Ivchenko, G. Karczewski, T. Wojtowicz, and J. Kossut, Spin Coherence of a Two-Dimensional Electron Gas Induced by Resonant Excitation of Trions and Excitons in CdTe/(Cd, Mg)Te Quantum Wells, Phys. Rev. B 76, 205310 (2007).

[35] I. A. Yugova, M. M. Glazov, E. L. Ivchenko, and Al. L. Efros, Pump-Probe Faraday Rotation and Ellipticity in an Ensemble of Singly Charged Quantum Dots, Phys. Rev. B 80, 104436 (2009).

[36] M. Cho, N. F. Scherer, G. R. Fleming, and S. Mukamel, Photon Echoes and Related Four-Wave-Mixing Spectroscopies Using Phase-Locked Pulses, J. Chem. Phys. 96, 5618 (1992).

[37] W. Langbein and P. Borri, in Semiconductor Qubits, edited by F. Henneberger and O. Benson (Pan Stanford, Singapore, 2008), p. 269.

[38] A. A. Sirenko, T. Ruf, M. Cardona, D. R. Yakovlev, W. Ossau, A. Waag, and G. Landwehr, Electron and Hole 
$g$ Factors Measured by Spin-Flip Raman Scattering in $\mathrm{CdTe} / \mathrm{Cd}_{1-x} \mathrm{Mg}_{x}$ Te Single Quantum Wells, Phys. Rev. B 56, 2114 (1997).

[39] A. R. Cameron, P. Riblet, and A. Miller, Spin Gratings and the Measurement of Electron Drift Mobility in Multiple Quantum Well Semiconductors, Phys. Rev. Lett. 76, 4793 (1996).
[40] S. G. Carter, Z. Chen, and S. T. Cundiff, Optical Measurement and Control of Spin Diffusion in n-Doped GaAs Quantum Wells, Phys. Rev. Lett. 97, 136602 (2006).

[41] G. Wang, B. L. Liu, A. Balocchi, P. Renucci, C. R. Zhu, T. Amand, C. Fontaine, and X. Marie, Gate Control of the Electron Spin-Diffusion Length in Semiconductor Quantum Wells, Nat. Commun. 4, 2372 (2013). 